\title{
Dielectric-technique-assisted breast cancer surgery
}

\author{
Marek Rząca ${ }^{1}$, Kazimierz Orzechowski ${ }^{2}$, Alicja Wolny² ${ }^{2}$ Magdalena Sitarska ${ }^{1}$
}

Introduction. In conservative breast cancer surgery, removing all carcinogenic lesions is a sound basis for achieving therapeutic success. The cleanness of the cutting margin is usually assessed after surgery and any residual cancerous tissue that are found, require further surgery. A simple and cheap assessment method is presented which can be used during surgery, the dielectric cancer probe (DCP), which allows the surgeon to take fast decisions on whether to extend incisions.

Method. The DCP method consists of measuring and then differentiating between the electrical properties (permeability and conductivity) of healthy and cancerous breast tissue. A polyethylene strip is used to separate the examined tissue from the instrument probe and the procedure is carried out by the operating surgeon.

Results. This method was found to allow correct identification of cancerous breast tissue based on taking 189 samples from 70 patients using the intraoperative hand probe. The sensitivity and specificity were respectively $87 \%$ and $99 \%$. Conclusions. The DCP is a high sensitivity method and is totally safe for the patient and can be used intraoperatively. The device is an aid to the surgeon for differentiating between healthy and cancerous breast tissue. Its widespread use should reduce the number of reoperations in the conservative surgical management of breast cancer patients.

NOWOTWORY J Oncol 2017; 67, 2: 108-114

Key words: breast cancer, breast conserving surgery, incision margins, dielectric probe, reoperation

\section{Introduction}

Breast cancer is the most common malignancy found in women aged 45-70 years in developed countries and is the most common cause of cancer related death [1].

There are several methods of treating breast cancer, however surgery remains the treatment of choice for cases of non-advanced disease. The aim of surgery is to secure clear resection margins. This can be achieved by mastectomy or breast conserving treatment (BCT). The success of local treatment depends on having clear resection margins. Residual cancer in resection margins can be found in as many as $20-46 \%$ of patients treated by BCT [2-4] which significantly increases the risk of recurrence, with ductal carcinoma in situ (DCIS) being particularly difficult to excise [5]. It is therefore vital to evaluate the clearance of excision margins. Routine examinations are undertaken by the histopathologist on samples harvested from the surface of the excised tissue [6]. This can of course be carried out intra- operatively, but since it is very time consuming it cannot be a standard approach. Such postoperative histopathology is accurate and has low false negativity, nevertheless its drawback is that hospital readmission and reoperation are required whenever cancerous tissue are found within the surgical involvement margins. There are also other methods of margin assessment available such as imprint cytology [7] and near IR fluorescence [8] or high frequency U/S [9].

The dielectric technique is a relatively new method [2, $10,11]$. It measures electric permittivity and conductivity which are known to differ between normal and malignant tissue [12-14]. These parameters are lowest in fat tissue and are significantly lower than in cancerous tissue $[15,16]$ thus allowing differentiation between these tissue states.

Laboratory testing has demonstrated such differences in clean and dried samples [17-19], however in real-life situations the presence of blood and serum interferes with such measurements by apparently increasing the permittivity

${ }^{1}$ Research and Development Centre, Voivodship Specialist Hospital in Wrocław, Poland

${ }^{2}$ Faculty of Chemistry, University of Wrocław, Poland 
and conductivity in normal tissue to those levels found in malignant tissue. For this reason the method is problematic for use during surgery [21, 22].

This study demonstrates the use of a probe with socalled blocked electrodes, where the electric sensor does not come into direct contact with the examined tissue but is separated by a thin layer of insulation (polyethylene film) [18-20] thus avoiding the conflicting interference from blood and serum. The insulating polyethylene between the probe and sample ensures sterility of the field of operation, thus allowing multiple uses of the same probe. This investigation aims to assess the value and reliability of the dielectric method in differentiating between normal from malignant tissue as well as whether it can be used for assessing the cleanness of the cutting margin during breast surgery and detecting any new foci of cancer (DTABCS - Dielectric Technique Assisted Breast Cancer Surgery). In particular, the study tests a prototype hand held probe, developed, manufactured and patented in Poland; the Dielectric Cancer Probe (DCP).

\section{Materials and methods}

Breast tissue samples were acquired at the time of surgery carried out on 70 patients at the Regional Specialist Hospital in Wroclaw from 01/01/2013 to 30/09/2015. We obtained 189 samples from 32 patients undergoing mastectomy and 34 patients having BCT; of these 4 patients had only local excisions for benign lumps.

Testing consisted of 94 normal breast tissue samples and 95 that were histopathologically confirmed as being malignant. Patient characteristics are shown in Table I. Samples were examined both intra-operatively and post-operatively. The standard sample was a thin sliver of tissue, harvested by the surgeon in such a way that it contained both normal and malignant tissue.

Obtaining samples for DCP was stand-alone and had no effect on those taken for the routine histopathological testing. DCP samples were stored in sealed containers at $5^{\circ} \mathrm{C}$ temperature and underwent dielectric testing within 24 hours since their acquisition. We confirmed such a time delay had no effect whatsoever on outcome (Fig. 1).

For the intra-operative tests, the tissue surface removed was checked for cleanliness and subsequently sent for histopathology. All measurements were carried out at room temperature $\left(20-25^{\circ} \mathrm{C}\right)$. Consent of the bioethics commission prior to the launch of this study had been obtained.

\section{Methods}

Figure 2 shows the DCP hand held probe. It is a Maxwell Wagner layer capacitor. Instrumental readings were related to the electric parameters of the tissue examined (i.e. permittivity and conductivity) and depend on the detector circuit and probe sensor geometry. The device is capable of obtain-
Table I. Patients' characteristics

\begin{tabular}{|c|c|}
\hline No of patients & 70 \\
\hline \multicolumn{2}{|l|}{ Age } \\
\hline The youngest & $25 y$ \\
\hline The oldest & $84 \mathrm{y}$ \\
\hline Average age & $60.7 y$ \\
\hline \multicolumn{2}{|l|}{ Preoperative diagnosis } \\
\hline Core biopsy & 66 \\
\hline No biopsy & 4 \\
\hline \multicolumn{2}{|l|}{ Histological type of tumour } \\
\hline Invasive ductal carcinoma & 46 \\
\hline Invasive ductal carcinoma with DCIS component & 10 \\
\hline Invasive lobular carcinoma & 8 \\
\hline DCIS & 1 \\
\hline Invasive metaplastic carcinoma & 1 \\
\hline Fibroadenoma & 4 \\
\hline \multicolumn{2}{|l|}{ Grading } \\
\hline G1 & 8 \\
\hline G2 & 38 \\
\hline G3 & 20 \\
\hline Angioinvasion & 29 \\
\hline N1 & 29 \\
\hline Preoperative chemotherapy & 5 \\
\hline \multicolumn{2}{|l|}{ Type of operation } \\
\hline Mastectomy & 32 \\
\hline Breast conserving surgery & 34 \\
\hline Lumpectomy & 4 \\
\hline \multicolumn{2}{|l|}{ Number of investigated specimens } \\
\hline Tumour tissue & 95 \\
\hline Healthy tissue & 94 \\
\hline
\end{tabular}

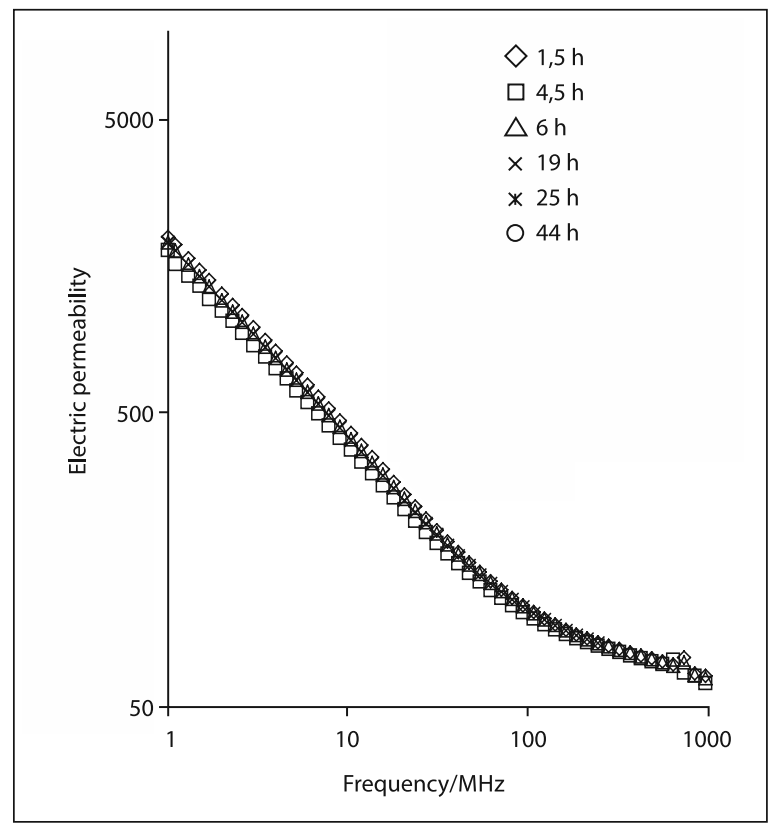

Figure 1. Dependence between sampling and measurement times. Curves are superimposed 


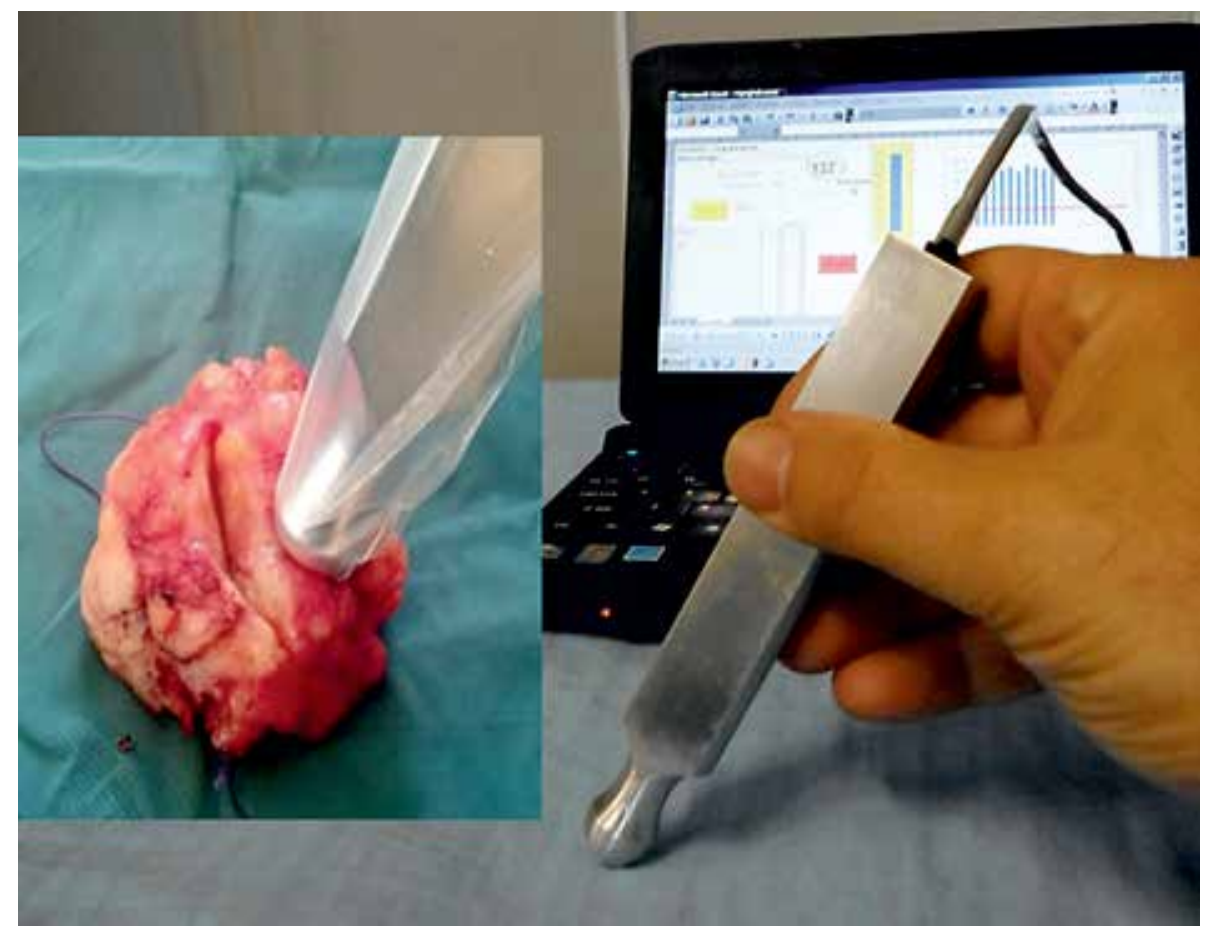

Figure 2. Prototype of the hand-held dielectric probe

ing readings ranging from 1 to 120 units depending on the type of tissue; with adipose tissue giving the lowest and cancer the highest. An operating $100 \mathrm{MHz}$ radio frequency was used which is harmless to patients. The probe has an oblong shape with a $25 \mathrm{~mm}^{2}$ sensor at its tip. Measurements are taken by pressing the probe against the tissue.

As aforementioned, a polyethylene sleeve was used to safeguard the sterility of the operations field and to eliminate the interference of blood and serum exudate. There was no effect demonstrated of the potentially interfering conductible fluids on the instrument results as shown on Figures $3 \mathrm{~A}$ and $3 \mathrm{~B}$; these showing the result of the test sample containing malignant tissue in the middle of the specimen. The Figure $3 \mathrm{~A}$ high readings confirm malignancy of a blood-free sample whilst Figure $3 B$ shows those of a sample dipped in normal saline.

Technical data and construction details of the DCP are provided in its patent description [17]. The physics back-

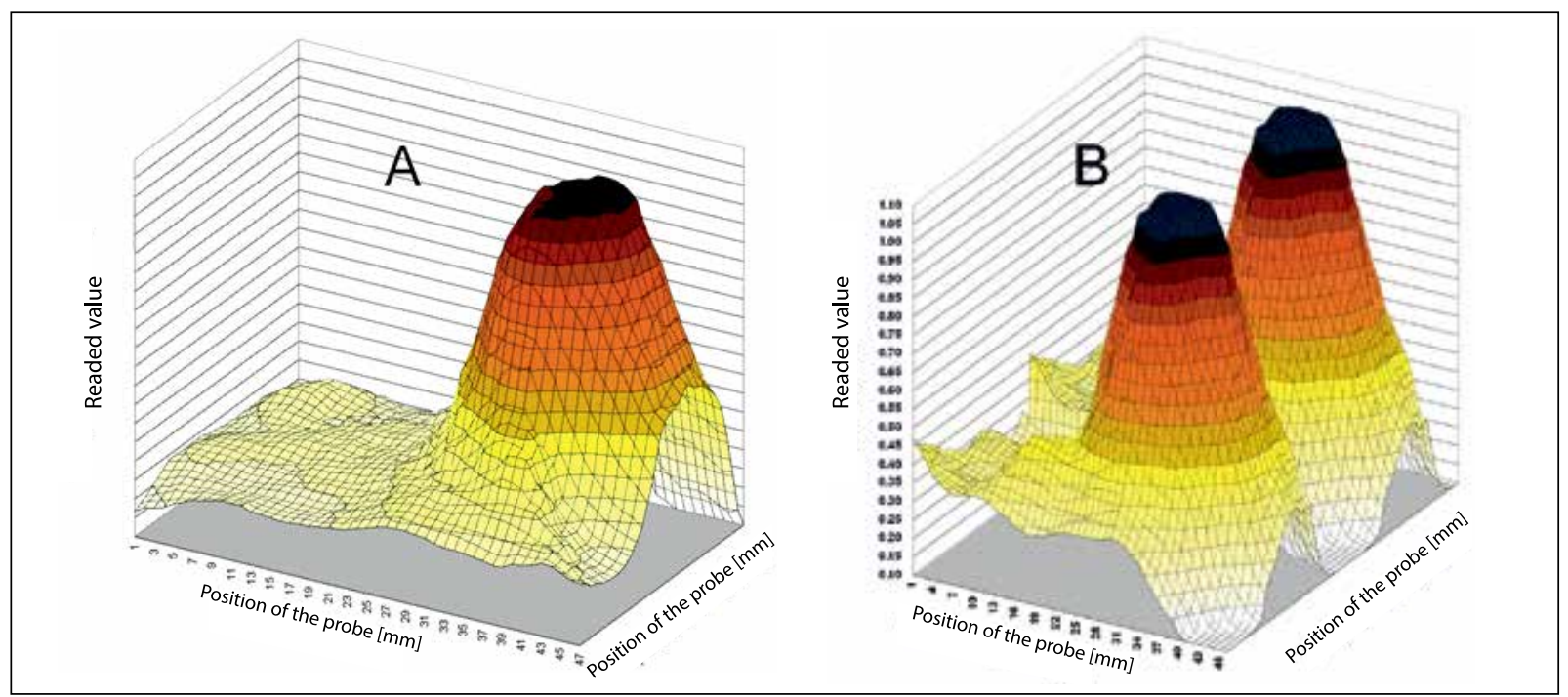

Figure 3. A dielectric map of measurements taken of a test sample containing malignant tissue in the middle of the specimen. High readings confirm malignancy of a blood-free sample (A) whilst a sample dipped in normal saline is shown in (B). No demonstrated effect of the conductible fluid on results 


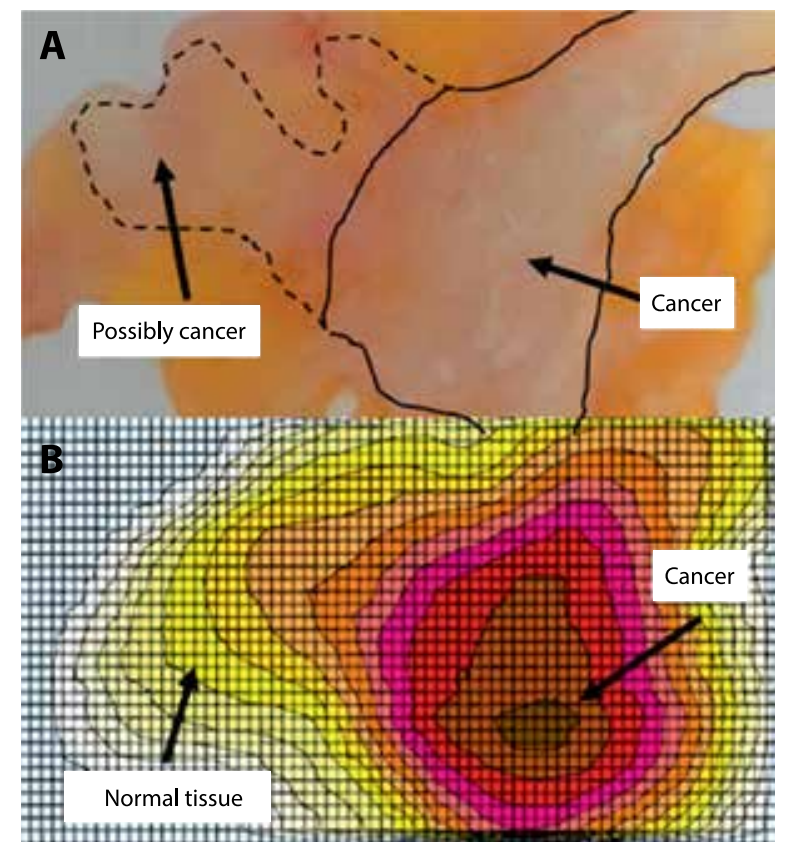

Figure 4. A - cross sectional image of a tissue sample containing neoplastic changes surrounded by healthy breast tissue, B - dielectric map

ground of the measurements and the tests carried out on reference substances and animal tissue have been described in earlier publications from physics journals [18-20].

Laboratory testing has demonstrated that cancer tissue can be detected both on the surface of a specimen as well as underneath a 1-2 mm layer of healthy breast tissue [18, 19].

The DCP is a simple to use and inexpensive device. It takes 6-12 minutes to examine the whole specimen. Results are displayed on a screen and the detection of malignant tissue triggers an audible alarm. The DCP is only a prototype and it is not yet commercially available. All our study tests were performed on prototypes manufactured by Mr Stanisław Baj (Zakład Nowych Technologii, Owczarska St. 42, 54-020 Wrocław, Poland; www.znt.com.pl).

\section{Results}

Figure 4 shows a cross sectional image of sample tissue (A) and a dielectric map (B) acquired by using the DCP during the test of its ability to detect neoplastic changes. The image shows a pinkish area corresponding to cancer. The dielectric map (B) shows brown and red areas of high permittivity and conductivity consistent with cancer, whereas the yellow and blue depict normal breast tissue. The close similarity between the images and dielectric maps are ex- plicitly visible. This study as well as earlier publications [19, 20] demonstrate multiple tests that have confirmed a correct differentiation between healthy and neoplastic tissue.

Statistical analysis showed for $100 \mathrm{MHz}$ frequency, that the dielectric probe correctly distinguishes healthy breast tissue from malignancy. Instrument readings varied from 2 to 36 units (mean 12) for normal breast tissue and 11 to 114 units (mean 58) for cancer. A non- parametric Kruskal Wallis test was used for the statistical analysis. For both sample groups (i.e. normal and malignant) the number of samples $(\mathrm{N})$, mean value $(\mathrm{X})$, median $(\mathrm{M})$, standard deviation (SD), range ( $\min$ - max), lower and upper quartyl (25Q-75Q) were calculated. A highly significant value $(p=0.0000)$ was obtained by the Kruskal-Wallis test; results being shown in Table II. The correlation between pathology and the results of dielectric testing was analysed to assess the sensitivity and specificity of the tested method based on 189 samples, of which 94 had benign histopathology whilst 95 were malignant. Figure 5 shows the ROC curve (Receiver Operating Characteristics).

The shape of the ROC curve demonstrates a high diagnostic potential for the tested method. The Youden Index (YI)

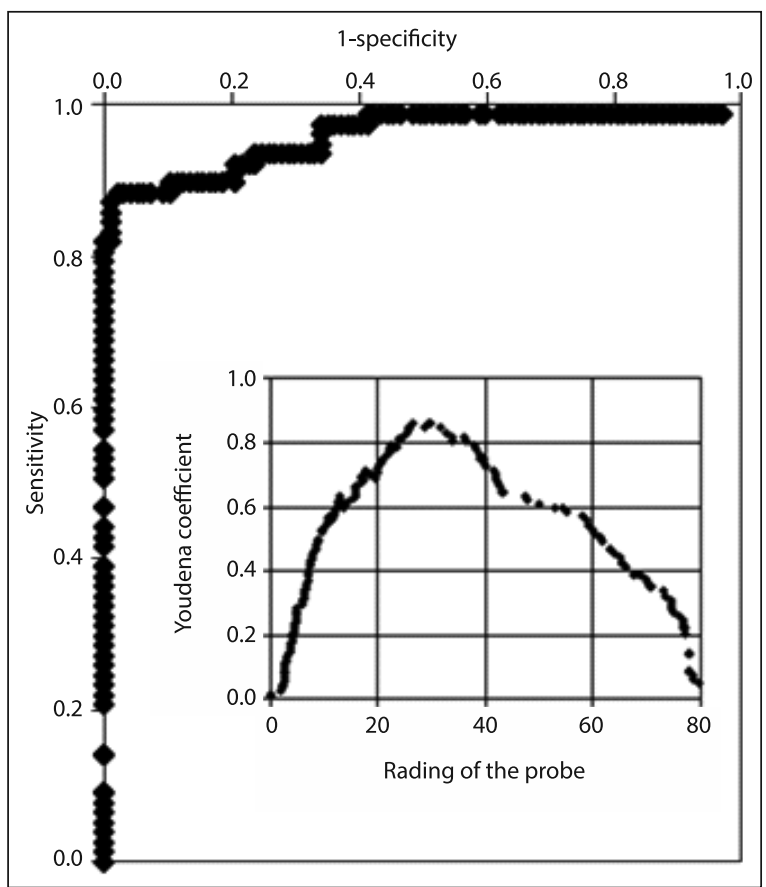

Figure 5. ROC curve. Correlation between sensitivity and specificity of the hand held DCP probe for detecting breast cancer

Table II. Results of the Kruskal-Wallis test

\begin{tabular}{lcccccccc}
\hline & $\mathrm{X}$ & $\mathrm{N}$ & $\mathrm{SD}$ & $\mathrm{MIN}$ & $\mathrm{MAX}$ & $\mathbf{2 5 Q}$ & $\mathrm{M}$ & $\mathbf{7 5 Q}$ \\
\hline Healthy tissue & 11.6 & 94 & 7.8 & 2.0 & 36.0 & 5.0 & 17.1 \\
Tumour & 58.8 & 95 & 21.6 & 11.3 & 114.1 & 41.5 & 65.2 & 77.1 \\
\hline
\end{tabular}




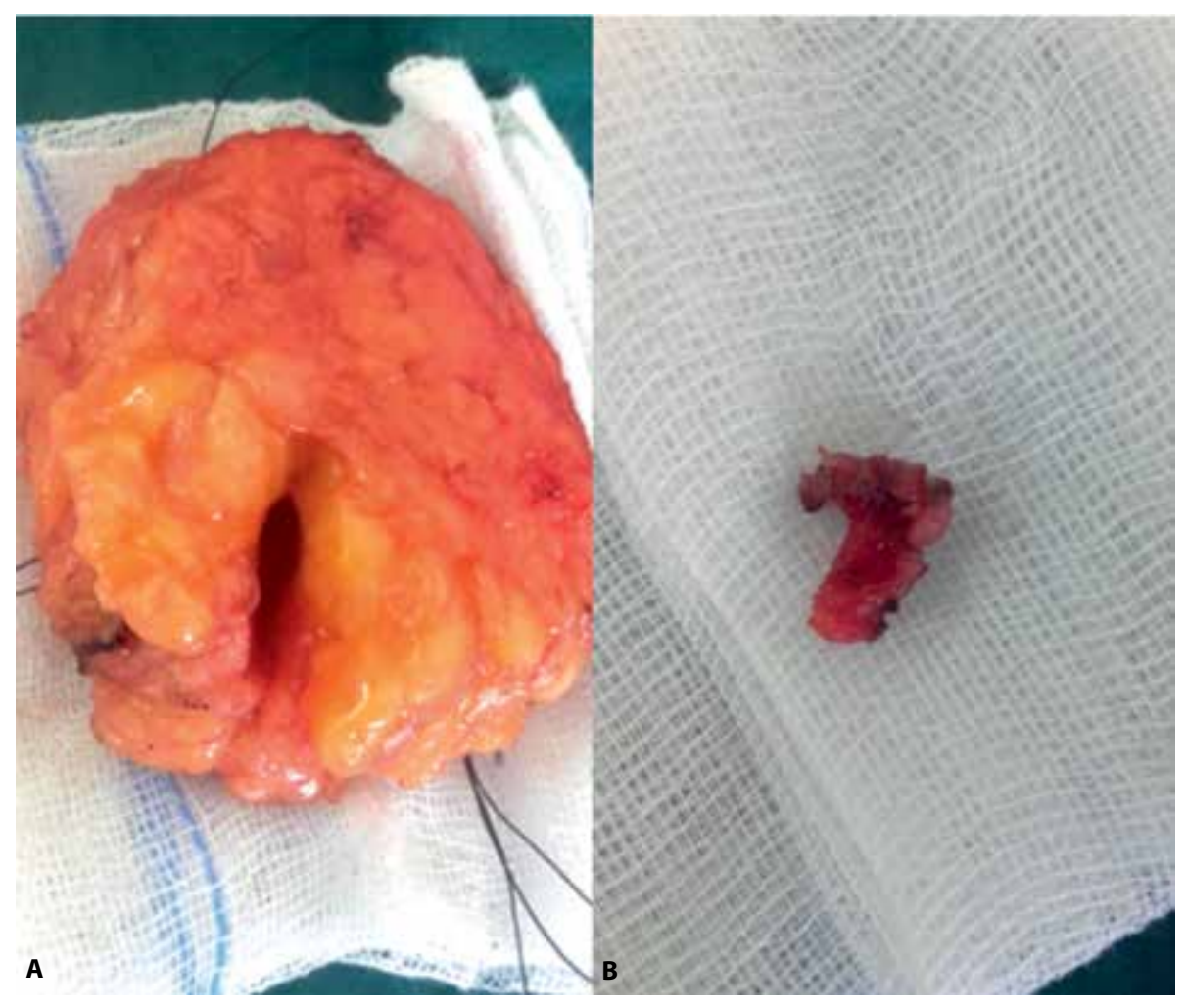

Figure 6. Pictures showing excised breast lump tissue (A) and an additional margin fragment (B). The difficulty posed to the pathologist is the lack of continuity of cancer infiltration and specimen fragmentation

was used to find the best cut off point which was calculated based on the sensitivity and specificity ( $\mathrm{YI}=$ sensitivity + specificity -1). The maximum of $\mathrm{Yl}$, i.e. the optimum cut off point was achieved for a reading of 30 units. This value is more than twice as high as the mean value for normal tissue. For the set cut off parameter, sensitivity and specificity were respectively 0.87 and 0.99 . These results place DCP among one of the best intra-operative techniques that are used to assess the clearance of margins. Our probe is capable of correctly diagnosing normal and neoplastic tissue, however it is unable to differentiate between benign and malignant neoplasms. Because of the physical parameters of such tissue, it is however unlikely that we will be able to ever achieve this goal.

Analysing false negatives and false positives results is necessary for evaluating any new diagnostic tool/method. We found only one false positive, where a sample found to be benign on histopathology was reported as being malignant by the probe. This was a NST G2, LV+ve, LN+ve cancer. We also noted 10 false negatives, among them 8 NST cancers and 2 lobular ones. Eight tumours were $G 2$ and two G3 stage. Six cases were LV+ve. Seven patients were treated with BCT and two received mastectomy. There was no correlation between false positives/negatives and pathological features such as grade, LV and tumour type. We suspect that an inadequate contact between the probe and the sample or perhaps a too thick layer of fatty tissue (greater than $3 \mathrm{~mm}$ ) could have been responsible.

\section{Discussion}

A significant number of patients who undergo $\mathrm{BCT}$ subsequently require additional surgery for the clearance of margins. In Poland there are approx. 18,000 new cases of breast cancer annually. If $50 \%$ of women undergo conservative treatment and $25 \%$ of these require further surgery, then we can assume that approx. 2,250 operations are performed annually to clear surgical margins after incomplete excision. The odds are even higher for impalpable and multifocal lesions and for DCIS. It is however difficult to unequivocally establish the involvement of cancerous margins by histopathology, where this is confirmed in only $63 \%$ cases of finding tumours in re-excision specimens [24]. Other studies have shown that cancer can still be found in margins wider than $2 \mathrm{~mm}$. This can be explained by a lack of continuity of cancer infiltration or by technical and specimen handling issues (Fig. 6) [25].

Intra-operative and postoperative histopathology can make mistakes. The intra-operative device in the hands of experienced surgeon can significantly reduce the need for repeating surgery by giving the surgeon the chance to excise wider margins at the initial operation. The probe of course cannot be a substitute for a rigorous histopatho- 
logical examination. In the literature there some reports of instruments using similar techniques to DCP. Dune Medical Devices, Caesarea, Israel, offers the MarginProbe ${ }^{\circledR}$. We would thereby like to include a review of their experience in order to compare their device with our DCP. In 2007, a study by T. Karni et al. intra-operatively assessed surgical margins using the MarginProbe ${ }^{\circledast}$ [26]. The sensitivity in detecting involved margins was $71 \%$ and the specificity was $68 \%$, depending on the width of the margin.

Pappo et al. [30] conducted a similar study on 753 samples obtained from 76 patients. Sensitivity and specificity reached $70 \%$, with the sensitivity increasing with the tumour size and reaching 97\% in lesions greater than $6 \mathrm{~mm}$ irrespective of the tumour type; our results having similar outcomes. A randomised trial by Thill et al was performed in 2011 and 2014 using the MarginProbe ${ }^{\circledR}$ on patients treated with BCT for DCIS which demonstrated reduced re-excision rates by $17 \%$. In $21 \%$ cases, mastectomy was performed as a one stage procedure thanks to this device $[10,11]$. In 2012, Riwera et al following recommendations of the FDA, conducted a multi-centre randomised trial which recruited 596 women with impalpable tumours. The use of MarginProbe ${ }^{\circledast}$ reduced the need for a second operation from $29.9 \%$ to $14.1 \%$. A similar trial by Schnabel et al [2] yielded excision rates of $62 \%$ for positive margins vs $22 \%$ without the probe. In summary, the total number of re-operations was reduced by $6 \%$. In 2015 , Sebastian et al performed a retrospective analysis of the data collected from three centres on 165 women subjects, where a $62 \%$ reduction in re-operations was achieved in those where the probe had been used [4].

According to all these studies, false positives and negatives were linked to small sample size and to the presence of blood and serum with high conductivity. Our DCP is however not sensitive to such circumstances.

As for now, the MarginProbe ${ }^{\circledast}$ has not been recommended by international authorities for treating breast cancer as a standard treatment in BCT $[28,29]$. This reserved and cautious judgment is due to insufficient data on the impact of this device on local recurrence. The recommendation may yet still be granted should more supporting data appear.

Just as in the case of the MarginProbe ${ }^{\circledast}$, our study did not also find any correlation between the probe reading and the tumour type. What is more important, there are no such differences in DCIS which is diagnosed successfully as invasive cancer. The MarginProbe ${ }^{\oplus}$ has been so far introduced mainly in Israel and the USA. There are some scanty reports also coming from Germany. The price of the console is $24,995 \$$ and the disposable probe costs 995 \$. These costs are significantly higher than the predicted price of the commercially available Polish DCP. Our probe is a multiple-use device which is capable of taking measurements in the presence of blood and serum within the field of operation.

\section{Conclusions}

This study has presented test results of the Dielectric Cancer Probe (DCP) developed and manufactured in Poland. This instrument is designed for intra-operatively differentiating between normal breast tissue from malignancy, and particularly for examining the clearance of surgical margins, as well as to search for residual cancer in the remaining post excision breast tissue. The background physics and how the method is used are similar to the commercially available MarginProbe ${ }^{\circledast}$ device. The advantage of our instrument over the latter is its insensitivity to the presence of blood and serum within the field of operation. The DCP is also a multiple use and inexpensive device which has no disposable parts requiring replacement after each test procedure. It is safe to use intra-operatively with a sterile polyethylene sleeve. The DCP is relatively inexpensive. Our study has demonstrated its efficiency in detecting involved surgical margins. The probe is however unable to distinguish benign from malignant tumours. The DCP accurately distinguishes and localises tissue that differs from normal breast parenchyma, not only on the surface of the specimen but also at a depth of 1-2 $\mathrm{mm}$. The DCP should therefore be treated as a surgeon's additional eye in helping to intra-operatively locate histopathologically abnormal tissue and thus aiding in taking the decision on whether any additional resection is required.

\section{Acknowledgement}

A.W. would like to thank the Department of Chemistry University of Wroclaw for their financial support (grant 1505/M/WCH15).

\section{Conflict of interest: none declared}

\author{
Marek Rząca, MD, PhD \\ Research and Development Centre \\ Voivodship Specialist Hospital in Wrocław \\ Kamieńskiego St. $73 a$ \\ 51-124 Wrocław, Polska \\ e-mail:marekrzaca@poczta.fm
}

Received: 30 Mar 2016

Accepted: 9 Feb 2017

\section{References}

1. Siegel R, Ma J, Zou Z et al. Cancer statistics, 2014. CA Cancer J Clin 2014; 64: 9-29.

2. Schnabel F, Boolbol S, Gittleman $M$ et al. A randomized prospective study of lumpectomy margin assessment with use of MarginProbe in patients with nonpalpable breast malignancies. Ann Surg Oncol 2014; 21: 1589-1595.

3. Landercasper J, Attai D, Atisha D et al. Toolbox to reduce lumpectomy reoperations and improve cosmetic outcome in breast cancer patients: The American Society of Breast Surgeons Consensus Conference. Ann Surg Oncol 2015; 22: 3174-3183.

4. Sebastian M, Akbari S, Anglin B et al. A. The impact of use of intraoperative margin assessment device on re-excision rates. Springerplus 2015; 4: 198. 
5. Dunne C, Burke JP, Morrow M et al. Effect of margin status on local recurrence after breast conservation and radiation therapy for ductal carcinoma in situ. J Clin Oncol. 2009; 27: 1615-1620.

6. Dener C, Inan A, Sen M et al. Intraoperative frozen section for margin assessment in breast conserving surgery. Scand J Surg 2009; 98: 34-40.

7. Klimberg VS, Harms S, Korourian S. Assessing margin status. Surg Oncol 1999; 8: 77-84.

8. Sevick-Muraca EM. Translation of near-infrared fluorescence imaging technologies: emerging clinical applications. Annu Rev Med 2012; 63: 217-231.

9. Doyle TE, Factor R, Ellefson C et al. High-frequency ultrasound for intraoperative margin assessments in breast conservation surgery: a feasibility study. BMC Cancer 2011; 11: 444.

10. Thill M, Röder K, Diedrich K et al. Intraoperative assessment of surgical margins during breast conserving surgery of ductal carcinoma in situ by use of radiofrequency spectroscopy. Breast 2011; 20: 579-580.

11. Thill M. MarginProbe : intraoperative margin assessment during breast conserving surgery by using radiofrequency spectroscopy. Expert Rev Med Devices 2013; 10: 301-315.

12. Lazebnik M, McCartney L, Popovic D et al. A large-scale study of the ultrawideband microwave dielectric properties of normal breast tissue obtained from reduction surgeries. Phys Med Biol 2007; 52: 2637-2656.

13. O'Rourke AP, Lazebnik M, Bertram JM et al. Dielectric properties of human normal, malignant and cirrhotic liver tissue: in vivo and ex vivo measurements from 0.5 to $20 \mathrm{GHz}$ using a precision open-ended coaxial probe. Phys Med Biol 2007; 52: 4707-4719.

14. Lukaszewicz K, Wtorek J, Bujnowski A et al. Monitoring of breast tissue thermo-ablation by means of impedance measurements. J Phys 2010 (Conf Ser. 224012136).

15. Gabriel S, Lau RW, Gabriel C. The dielectric properties of biological tissues: II. Measurements in the frequency range $10 \mathrm{~Hz}$ to $20 \mathrm{GHz}$. Phys Med Biol 1996; 41: 2251-2269.

16. Foster K, Schwan HP. Dielectric properties of tissues and biological materials: a critical review. Crit Rev Biomed Eng 1989; 17: 25-104.

17. Orzechowski K, Rząca M, Baj S. Probe for localization of cancer tissue, Polish patent No 217421

18. Orzechowski K, Rudowski M, Rząca M. Test of the method of differentiation between healthy and anomalous breast tissues using the open-ended dielectric probe covered by a dielectric film. Physiol Meas 2008; 29: S321-330.

19. Rudowski M, Orzechowski K, Rząca M. Modified open-ended dielectric probe in distinction between cancer and healthy breast tissues. J NonCryst Solids 2010; 356: 730-732.

20. Wolny A, Orzechowski K, Rudowski M. Measurements of electric properties using an open-ended probe blocked by a non-conducting foil. Results in Physics 2016; 6: 288-292.

21. Grant EH, Sheppard RJ, South SP. Dielectric behavior of biological molecules in solution. Oxford; London: Oxford Universty Press, 1978.

22. Ishai PB, Talary MS, Caduff A et al. Electrode polarization in dielectric measurements: a review. Meas Sci Technol 2013; 24: 102001.

23. Rząca M. Wykorzystanie kliniczne spektroskopii mikrofalowej do rozpoznawania zmian nowotworowych gruczołu piersiowego. PhD Thesis. Akademia Medyczna we Wrocławiu, 2009.

24. Scopa CD, Aroukatos P, Tsamandas AC et al. Evaluation of margin status in lumpectomy specimens and residual breast carcinoma. Breast $J$ 2006; 12: 150-153.

25. Skripanowa S, Layfield LJ. Initial margin status for invasive ductal carcinoma of the breast and subsequent identification of carcinoma in reexcision specimens. Arch Pathol Lab Med 2010; 134: 109-114.

26. Karni T, Pappo I, Sandbank J et al. A device for real-time, intraoperative margin assessment in breast-conservation surgery. Am J Surg 2007; 194: 467-473.

27. Rivera RJ, Holmes DR, Tafra L. Analysis of the impact of intraoperative margin assessment with adjunctive use of margin probe versus standard of care on tissue volume removed. Int J Surg Oncol 2012; 2012: 868623. doi:10.1155/2012/868623.

28. Health Policy Advisory Committee on Technology. Margin Probe to assess surgical margin during lumpectomy for breast cancer. February 3013. https:// www.health.qld.gov.au/_data/assets/pdf_file/0024/427182/wp141.pdf.

29. Intraoperative assessment of surgical margins during breast-conserving surgery with radiofrequency spectroscopy or optical coherence tomography. https://medicalpolicy.simplyhealthcareplans.com/medicalpolicies/policies/mp pw c171884.htm.

30. Pappol, Spector R, Schindel A et al. Diagnostic performance of a novel device for real-time margin assessment in lumpectomy specimens. J Surg Res 2010; 160: 277-281. 